\title{
Sexual dimorphism in transmission of expression of islet autoantibodies to offspring
}

\author{
L. Yu ${ }^{1}$, H.P. Chase ${ }^{1}$, A. Falorni ${ }^{2}$, M.Rewers ${ }^{3}$, A. Lermmark ${ }^{2}$, G. S. Eisenbarth ${ }^{1}$ \\ ${ }^{1}$ Barbara Davis Center for Childhood Diabetes, University of Colorado Health Sciences Center, Denver, Colorado, USA \\ ${ }^{2}$ Department of Molecular Medicine, Karolinska Institute, Stockholm, Sweden \\ ${ }^{3}$ Department of Preventive Medicine, University of Colorado Health Sciences Center, Denver, Colorado, USA
}

Summary To help elucidate the mode of inheritance of insulin-dependent diabetes mellitus (IDDM), we measured GAD (glutamic acid decarboxylase) autoantibodies (GAD65 Ab), insulin autoantibodies (IAA), and cytoplasmic islet cell autoantibodies (ICA) in 292 sequentially screened non-diabetic offspring of patients with IDDM. The prevalence of these islet autoantibodies was higher in offspring of diabetic fathers than in offspring of diabetic mothers. The prevalences of GAD65Ab, IAA, and ICA in the offspring of diabetic fathers were $11.5 \%, 10.8 \%$, and $8.1 \%$ vs $2.1 \%, 1.4 \%$, and $2.8 \%$, respectively in the offspring of diabetic mothers $(p<0.002$, $p<0.001$, and $p=0.06 \mathrm{NS}$ ). Amongst autoantibodypositive relatives the IAA and ICA levels were significantly higher in offspring of diabetic fathers than of diabetic mothers $(p<0.002$ and $p<0.01$, respectively). The frequencies of these autoantibodies were equal in male and female offspring. We conclude that IDDM mothers transmitted islet autoimmunity less frequently to their offspring than IDDM fathers. Given the markedly lower frequency of autoantibodies in offspring of mothers, larger sample sizes will be required to determine whether islet autoantibodies are influenced by age of IDDM onset of mothers, maternal age of pregnancy, and presence of diabetes in these mothers prior to conception. [Diabetologia (1995) 38: 1353-1357]

Key words Islet autoantibodies, offspring, autoimmunity, transmission.
There is general agreement that offspring of mothers with insulin-dependent diabetes mellitus (IDDM) are at lower risk of diabetes compared to offspring of fathers with IDDM [1-3]. The lower risk has not been explained by classic mechanisms for producing a sex difference in transmission of a genetic trait because males and females are similarly

Received: 19 December 1994 and in revised form: 15 May 1995

Corresponding author: Dr. G.S.Eisenbarth, Barbara Davis Center for Childhood Diabetes, Box B140, University of Colorado Health Sciences Center, 4200 East 9 th Avenue, Denver, CO 80262, USA

Abbreviations: IDDM, Insulin-dependent diabetes mellitus; GAD, glutamic acid decarboxylase; GAD65Ab, glutamic acid decarboxylase autoantibodies; IAA, insulin autoantibodies; ICA, cytoplasmic islet cell autoantibodies; JDF, Juvenile Diabetes Foundation. affected by IDDM [1] and the risk for the offspring of mothers with a young age at onset of diabetes ( $<10$ years) may be similar to that for offspring of fathers with IDDM [3]. Furthermore, there is no clear evidence that the lower risk can be attributed to selective loss of the susceptible phenotype through perinatal death or spontaneous abortions [4].

In order to better understand this phenomenon, we assessed humoral autoimmunity to islets in nondiabetic offspring of IDDM fathers or mothers by analysing multiple islet autoantibodies including GAD (glutamic acid decarboxylase) autoantibodies (GAD65Ab), insulin autoantibodies (IAA), and cytoplasmic islet cell autoantibodies (ICA). An understanding of sex difference in IDDM transmission will provide important clues for the prediction and prevention of IDDM. 


\section{Subjects and methods}

Serum samples from 205 families (as part of a larger study determining the presence of islet autoantibodies of first-degree relatives of patients with IDDM) were collected at the-Barbara Davis Center from 292 non-diabetic offspring with only one parent with IDDM (median age 11.0 years). These samples represented the initial screening sample of the offspring. These samples were aliquoted and stored at $-20^{\circ} \mathrm{C}$. At the time of screening the 148 offspring of 103 diabetic fathers age $16 \pm 12$ years (mean $\pm S D$ ) and the 144 offspring of 102 diabetic mothers had a mean age of $15 \pm 11$ years. In addition, sera from 205 healthy control subjects without a family history of diabetes were analysed for GAD65Ab and LAA levels. The upper limits of the normal ranges for GAD65Ab and IAA were based on the 99th percentile of these healthy control samples.

IAA radioassay. IAA was determined with a competitive radioimmunoassay as previously described [5]. Radiolabelled recombinant human insulin ( ${ }^{125}$ I-Insulin, Amersham) was used in an immunoprecipitation assay with $150 \mu \mathrm{l}$ of patient serum at one third dilution with duplicates (with and without unlabelled human insulin for competition). The 99th percentile of normal range is $42 \mathrm{nU} / \mathrm{ml}$. The interassay coefficient of variation is $10.3 \%(n=7)$. In the 1993 and 1994 IAA proficiency tests, organized by the University of Florida, both the sensitivity and the specificity were $100 \%$ for both years.

$I C A$ assay. ICA was detected in patient sera by indirect immunofluoresence on unfixed cryostat sections of human pancreas. Juvenile Diabetes Foundation (JDF) units were determined using dilution of sera in parallel with standard samples and because of its higher risk for IDDM $\geq 20$ JDF units was defined as positive. In the ICA proficiency tests since 1992, organized by the University of Florida, the mean sensitivity of our assay was $86 \%$ and mean specificity was $100 \%$.

GAD65Ab radioassay. GAD65 was determined by a previously described radiobinding assay [6] with the following modifications. Radiolabelled recombinant human GAD65 $\left({ }^{35} \mathrm{~S}\right.$ GAD65) was produced by in vitro coupled transcription/translation of cDNA, and used in an immunoprecipitation assay with human serum at a $1 / 25$ dilution with triplicates for each sample. Antibody-bound ${ }^{35} \mathrm{~S}-\mathrm{GAD} 65$ was separated from free antigen by protein A-Sepharose. Immunoprecipitated radioactivity was determined and the antibody levels expressed as a relative index [index $=($ mean $\mathrm{cpm}$ of a sample $\mathrm{cpm}-$ mean cpm of three negative control samples)/(mean cpm of a positive control subject - mean cpm of three negative control subjects)]. Sera from one positive patient and three healthy subjects served as positive and negative standards, separately. The interassay coefficient of variation is $6.5 \%(n=10)$ and the 99 th percentile of normal range (index) is 0.032 . Samples with an index above 0.8 were further tested at $1 / 100$ and $1 / 250$ dilutions. In the 2nd International GAD Autoantibody Workshop, the GAD65Ab assay showed $87 \%$ sensitivity and $91 \%$ specificity. Adjusted sensitivity was $82 \%$ after adjustment of specificity to $100 \%$.

\section{Statistical analysis}

Statistical analysis was performed using the chi-square test and Fisher's exact test with Epistat (Richardson, Texas, USA) software.

\section{Results}

The levels and frequencies of the three islet autoantibodies in offspring of IDDM fathers and mothers are shown in Figure 1. GAD65Ab, IAA, and ICA were detected in $11.5 \%$ (17 of 148), $10.8 \%$ (16 of 148 ), and $8.1 \%$ (12 of 148$)$ of the offspring of diabetic fathers compared to $2.1 \%$ ( 3 of 144 ), $1.4 \%$ ( 2 of 144 ), and $2.8 \%$ ( 4 of 144$)$ of the offspring of diabetic mothers $(p<0.002,0.001$, and $p=0.06 \mathrm{NS}$, respectively). Relatives with increased levels of IAA and titres of ICA are at greater risk of developing diabetes [5, 7]. As shown in Figure $1 \mathrm{~b}$, none of the offspring of diabetic mothers had IAA levels above $80 \mathrm{nU} / \mathrm{ml}$, a level reported to be associated with both long-term persistence of such antibodies and increased progression to overt diabetes [5]. In contrast, 10 of 16 IAA-positive offspring of diabetic fathers had IAA above $80 \mathrm{nU} / \mathrm{ml}(p<0.002)$. Of four ICA-positive offspring of diabetic mothers none had a titre greater than $20 \mathrm{JDF}$ units (Fig. 1c), while 8 of 12 ICA-positive offspring of diabetic fathers had high titres of ICA ( $\geq 40$ JDF units; $p<0.01$ ).

Relatives expressing multiple islet autoantibodies rather than a single antibody are likely to be at higher risk of developing IDDM [8, 9]. Figure 2 illustrates the frequencies of combinations of the three islet autoantibodies (GAD65Ab, IAA, and ICA) in the offspring of diabetic fathers and mothers. Eight offspring of diabetic mothers expressed one autoantibody, while only one of them expressed two antibodies, and none expressed three. This compares with 31 offspring of diabetic fathers who expressed one or more autoantibodies, 9 of whom expressed two autoantibodies, and 5 three autoantibodies $(p<0.0002$, $p<0.02$, and $p=0.06 \mathrm{NS}$, respectively).

The appearance of any one of these three islet autoantibodies was associated with a higher likelihood of the appearance of a second autoantibody in the offspring of diabetic fathers as illustrated in Table 1 (the number of positive offspring of diabetic mothers was insufficient to permit analysis). For offspring positive for any of the three autoantibodies $47-58 \%$ expressed another autoantibody while only $11-15 \%$ of relatives negative for any one of the antibodies were positive for another antibody $(p<0.001)$.

There was no relationship between the age of diabetes onset of fathers and the appearance of these islet autoantibodies in their offspring (age $21 \pm 9$ years at onset of diabetes for the fathers of autoantibodypositive offspring compared to age $22 \pm 10$ years at onset for the fathers of autoantibody-negative offspring). The frequencies of the islet autoantibodies $(\geq 1 \mathrm{Ab})$ were equal in male and female offspring of diabetic fathers (16 of 64 male offspring were positive, compared to 16 of 84 female offspring, $p=0.4$ ). Larger sample sizes will be required to analyze for the effects of age of IDDM onset of mothers, maternal 

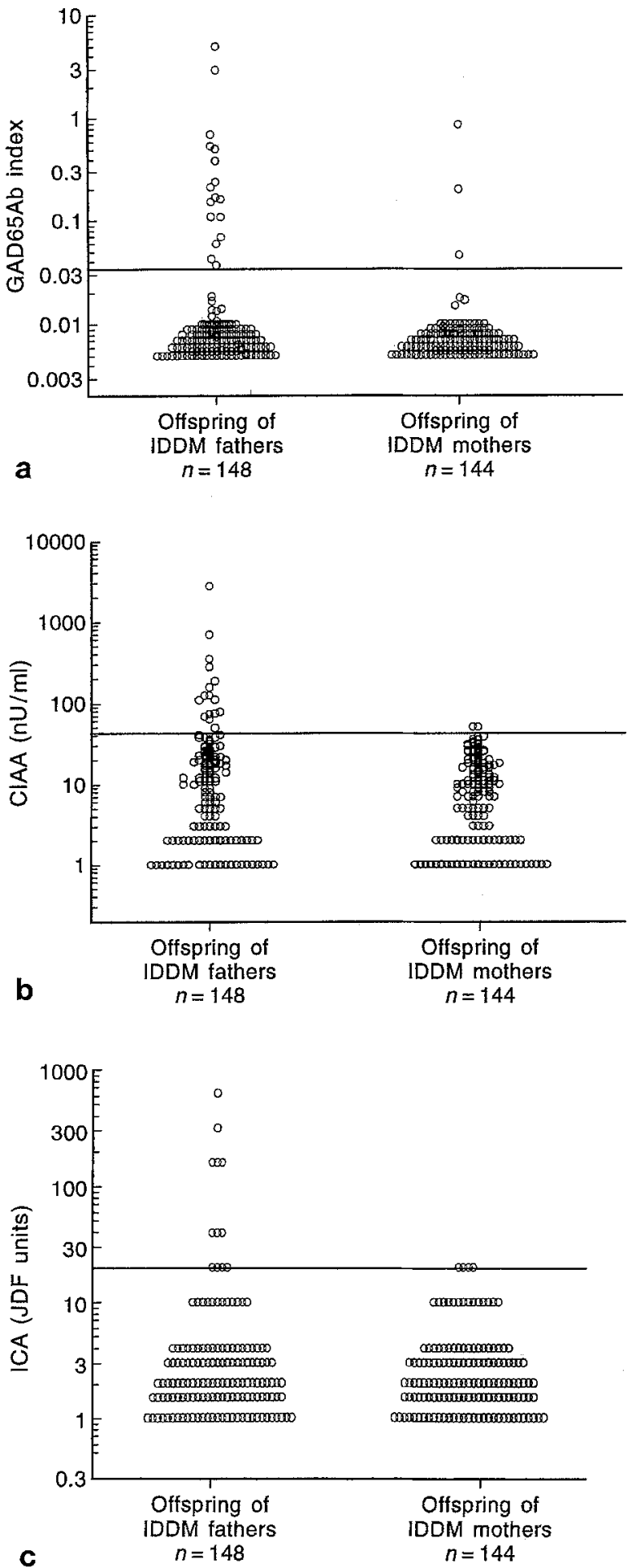

Fig. 1 (a-c). Levels of islet autoantibodies in 148 offspring of IDDM fathers and 144 offspring of IDDM mothers. (a) GAD65Ab index in the offspring of diabetic fathers and mothers. For frequency of positive, $p<0,002$. (b) IAA levels (nU/ $\mathrm{ml}$ ) in these offspring. For frequency of positive, $p<0.001$. (c) ICA JDF units in these offspring. For frequency of positive, $p=0.06 \mathrm{NS}$

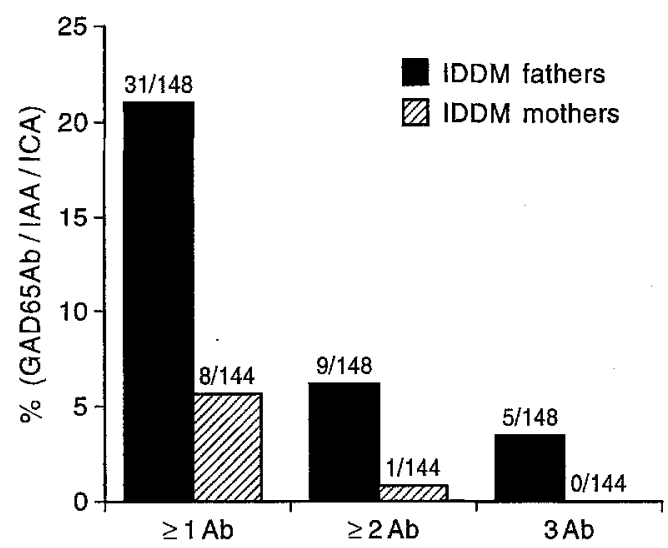

Fig. 2. The frequencies of combinations of three autoantibodies (GAD65Ab, IAA, ICA) in 148 offspring of IDDM fathers and 144 offspring of IDDM mothers. $p<0.0002$, $p=0.02, p=0.06$ respectively for offspring with $\geq 1 \mathrm{Ab}$, $\geq 2 \mathrm{Ab}, 3 \mathrm{Ab}$

age of pregnancy, and presence or absence of IDDM in the mothers at the time of pregnancy. With only eight offspring expressing any autoantibody, all IDDM-onset ages of the mothers were between 11 and 24 years.

\section{Discussion}

The risk of IDDM in offspring of diabetic fathers is 2 to 3 times greater than in offspring of diabetic mothers [1-3]. In the present study, we demonstrate that the offspring of diabetic mothers have significantly less anti-islet humoral autoimmunity, indicating that diabetic mothers transmit such islet autoimmunity to their offspring less frequently than diabetic fathers. The clinical follow-up for this group of offspring is currently very short (median 2 years) and only one offspring from the group with IDDM fathers expressing all three autoantibodies has progressed to overt IDDM. The differential expression of radioassay-determined autoantibodies by offspring of fathers with IDDM vs mothers with IDDM was particularly striking. Five times more offspring of IDDM fathers expressed either GAD65Ab or IAA. In a concordant manner, almost nine times more offspring of IDDM fathers expressed two or more autoantibodies as compared to offspring of IDDM mothers (6.1 vs $0.7 \%$ ). This excess of autoantibodies in offspring of fathers was evident already in the youngest cohort studies (e.g., in those offspring aged $<5$ years, 4 of $16(25 \%)$ of offspring of IDDM fathers expressed autoantibodies as compared to 0 of 10 similar-age offspring of IDDM mothers expressing any autoantibodies).

There are several hypotheses which have been proposed to explain the lower transmission of IDDM from diabetic mothers to their offspring. War- 
Table 1. Correlation of three islet autoantibodies in offspring of IDDM fathers

\begin{tabular}{llllrlr}
\hline & GAD65Ab+ & GAD65Ab- & IAA+ & IAA- & ICA+ & ICA- \\
\hline 1 other Ab+ & 3 & 13 & 3 & 14 & 2 & 16 \\
2 other Ab+ & 5 & 1 & 5 & 1 & 5 & 3 \\
2 other Ab- & 9 & 117 & 8 & 117 & 5 & 117 \\
\hline$p$ & $<0.007$ & & & $<0.007$ & & $<0.007$ \\
\hline
\end{tabular}

ram et al. [4] suggested that exposure in utero to an affected mother may induce immunologic tolerance to the islet autoantigens and thus protect a fetus from developing IDDM later in life. Recently, Bleich et al. [3] reported that mothers who were already diabetic when they gave birth did not transmit diabetes to their offspring at a different rate than mothers who developed diabetes after the birth of their children, which suggested that the difference in transmission rate of IDDM is not primarily due to a uterine diabetic milieu. Their data also indicated that there was no difference in diabetes transmission due to the duration of maternal diabetes at the time of delivery, which also argues against a maternal diabetic environment as a contributing factor. Finally, they found that the sex difference in transmission rate of IDDM to offspring may be due to a decrease in transmission rate of diabetes from mothers who develop diabetes after age 10 years [3]. Such an influence of the mothers' age at time of diabetes diagnosis is controversial [10]. The present study shows that the frequencies of these autoantibodies in offspring of IDDM mothers were influenced by the age of IDDM-onset of mothers. Finally, Warram et al. [11] reported that risk of IDDM in children of diabetic mothers decreases with increasing maternal age at pregnancy. In their study, a child born to a woman more than 25 years old with IDDM had a lower risk of developing IDDM than a child born to a younger mother. As the numbers of islet autoantibody-positive offspring of diabetic mothers in the current study are very small, we cannot yet perform subset analyses to see if the above age-related factors influence the prevalence of islet autoantibodies in offspring of diabetic mothers.

Genetic imprinting might be responsible for less frequent expression of diabetogenic genes amongst offspring of diabetic mothers. Recently, Julier et al. [12] reported data consistent with maternal imprinting of a 19-kilobase region of insulin-like growth factor-2 associated with IDDM risk. A study from Great Britain did not find evidence for imprinting of IDDM associated with insulin alleles [13], while our studies in the United States provided evidence for such imprinting [14]. In these studies, inheritance of a B FOK allele of the insulin gene appeared to protect from IDDM only upon paternal transmission. Thus, such "imprinting" by mothers of the potentially "protective" insulin gene is contradictory to the current observations of a higher transmission of IDDM and anti-islet autoantibodies from diabetic fathers compared to diabetic mothers.

While the mechanism underlying the higher prevalence of IDDM in offspring of diabetic fathers remains to be elucidated, we have demonstrated in this study that the prevalence of anti-islet humoral autoimmunity is much higher in offspring of diabetic fathers than in offspring of diabetic mothers. Evaluation of larger numbers of offspring of diabetic mothers should be carried out to explore the mechanism of the differing transmission rates. It will be of interest to determine whether prevalence of autoantibodies is influenced by whether the mother is diabetic at the time of pregnancy, age of diabetes onset of the mother, and age of pregnancy. The current finding of a fivefold difference in expression of autoantibodies, coupled with a much higher prevalence of autoantibodies relative to overt diabetes, should greatly facilitate analysis of the above questions. In particular, autoantibody expression often begins early in life, while development of diabetes is delayed by years. We believe that an understanding of the differential transmission of anti-islet autoimmunity, if of environmental origin, may suggest specific preventive therapies (e.g., insulin immunization) and, if of genetic origin, will help elucidate the underlying cause of IDDM.

Acknowledgements. We thank D. Dunlop for secretarial assistance. This work was supported by NIH grants DK32083, DK 43279, DK 32493, and DK 42654, and grants from BlumKovler Foundation, the Juvenile Diabetes Foundation, the Children's Diabetes Foundation at Denver, the Swedish Medical Research Council, and the Swedish Diabetes Association. Dr. A. Falorni was supported by a JDFI fellowship.

\section{References}

1. Warram JH, Krolewski AS, Gottlieb MS, Kahn CR (1984) Differences in risk of insulin-dependent diabetes in offspring of diabetic mothers and diabetic fathers. N Engl J Med 311: 3: 149-152

2. Tillil H, Kobberling J (1987) Age-correlated empirical genetic risk estimates for first degree relatives of IDDM patients. Diabetes 36:93-99

3. Bleich D, Polak M, Eisenbarth GS, Jackson RA (1993) Decreased risk of type I diabetes in offspring of mothers who acquire diabetes during adrenarchy. Diabetes 42: 14331439 
4. Warram JH, Krolewski AS, Kahn CR (1988) Determinants of IDDM and perinatal mortality in children of diabetic mothers. Diabetes 37: 1328-1334

5. Ziegler AG, Ziegler R, Vardi P, Jackson RA, Soeldner JS, Eisenbarth GS (1989) Life table analysis of progression to diabetes of anti-insulin autoantibody positive relatives of individuals with type I diabetes. Diabetes 38: 1320-1325

6. Falorni A, Grubin CE, Takei I et al. (1994) Radioimmunoassay detects the frequent occurrence of autoantibodies to the $M_{r} 65,000$ isoform of glutamic acid decarboxylase in Japanese insulin-dependent diabetes. Autoimmunity 19: 113-125

7. Bonifacio E, Bingley PJ, Shattock M et al. (1990) Quantitative islet cell antibody measurement assists in the prediction of insulin-dependent diabetes. Lancet 335: 147-149

8. Pietropaolo M, Yu L, Eisenbarth GS (1994) Biochemical prediction and diagnosis of type I diabetes. Clin Res 42: 117 A (Abstract)

9. Bingley PJ, Christie MR, Bonifacio E et al. (1994) Combined analysis of autoantibodies improves prediction of IDDM in islet cell antibody-positive relatives. Diabetes 43: $1304-1310$
10. Warram JH, Rich SS, Krolewski AS (1994) Epidemiology and genetics of diabetes mellitus. In: Kahn CR, Weir GC (eds) Joslin's Diabetes Mellitus. 13th edn. Lea \& Febiger, Philadelphia, pp 201-215

11. Warram JH, Blaise CM, Krolewski AS (1991) Risk of IDDM in children of diabetic mothers decreases with increasing maternal age at pregnancy. Diabetes 40: 16791684

12. Julier C, Hyer RN, Davies J et al. (1991) Insulin-IGF2 region on chromosome 11p encodes a gene implicated in HLA-DR4-dependent diabetes susceptibility. Nature 354: 155-159

13. Bain SC, Prins JB, Hearne CM et al. (1992) Insulin gene region-encoded susceptibility to type I diabetes is not restricted to HLA-DR4-positive individuals. Nature Genet 2: $212-215$

14. Pugliese A, Awdeh ZL, Alper CA, Jackson RA, Eisenbarth GS (1994) The paternally inherited insulin gene B allele (1,428 FokI site) confers protection from insulin-dependent diabetes in families. J Autoimmunity 7: 687-694 